Check for updates

Cite this: Mater. Adv., 2021, 2,3088

Received 9th February 2021 Accepted 30th March 2021

DOI: $10.1039 / \mathrm{d} 1 \mathrm{ma} 00119 \mathrm{a}$

rsc.li/materials-advances

\section{A versatile platform of poly(acrylic acid) cryogel for highly efficient photothermal water evaporation $\uparrow$}

\author{
Jia-Yun Wang, ${ }^{a}$ Xiao-Xiao Guo, ${ }^{a}$ Jun Chen, ${ }^{a}$ Shi-Chang Hou, ${ }^{a}$ Hui-Juan Li, ${ }^{a}$ \\ Abdul Haleem, ${ }^{a}$ Sheng-Qi Chen (DD ${ }^{b}$ and Wei-Dong He (D) *a
}

\begin{abstract}
Photothermal water evaporation, benefiting from solar energy, is one hot topic concerning seawater desalination, sewage treatment, and water recovery. Commonly, the photothermal evaporation system consists of photothermal material supported by certain matrices with fundamental necessities. Poly(acrylic acid) (PAAc) cryogels, prepared at the temperature below the freezing point of polymerization mixture, are hierarchically macro-porous and highly hydrophilic with rapid water uptake, speedy water transport, and low thermal conductivity. Moreover, PAAc has the acid-doping ability for polyaniline and polypyrrole (PPy), which faciliates the good incorporation of photothermal materials in a cryogel matrix. Thus, the PAAc cryogel is an excellent platform for photothermal water evaporation. In this research, we prepared PAAc cryogels, incorporated different photothermal materials, and constructed the modular water evaporation systems. The photothermal evaporation rate of pure water with the PAAc-PPy cryogel system reached as high as $1.819 \mathrm{~kg} \mathrm{~m}^{-2} \mathrm{~h}^{-1}$ while its apparent efficiency surprisingly exceeded $100 \%$. These modular systems exhibited excellent long-term and recyclable utility. As for the photothermal evaporation of salty water, the precipitated salt solid could be spontaneously dissolved during the light turnoff due to the quick water transport, maintaining the high water-to-vapor conversion. This study represents an ideal matrix platform, PAAc cryogel, for photothermal evaporation and opens up a new route to improve the photothermal evaporation technique for water purification and seawater desalination.
\end{abstract}

\section{Introduction}

The global area covered by water is as high as $70.8 \%$ but the freshwater resource is extremely limited and unevenly located. Of the total water resource, $99.7 \%$ is salty and polluted water, which is unfit for direct consumption. ${ }^{1,2}$ Therefore, techniques and materials for environment-friendly and efficient wastewater purification and seawater desalination are urgently needed. Solar energy is a green, inexhaustible, and recyclable resource. With the assistance of photothermal materials, solar energy is converted into heat for water evaporation, offering a green and promising route in water treatment. ${ }^{3-5}$ To attain highly efficient photothermal evaporation performance, there

\footnotetext{
${ }^{a}$ CAS Key Laboratory of Soft Matter Chemistry, Department of Polymer Science and Engineering, University of Science and Technology of China, Hefei, Anhui 230026, China.E-mail:wdhe@ustc.edu.cn

${ }^{b}$ Key Laboratory of Xin'an Medicine, Ministry of Education; Engineering Technology Research Center of Modernized Pharmaceutics, Anhui Province; Anhui University of Chinese Medicine, Hefei, Anhui 230038, China

$\dagger$ Electronic supplementary information (ESI) available. See DOI: 10.1039/ d1ma00119a
}

still exist many challenges to meet, including the photothermal material with excellent photo-physical properties and the evaporation system with high mass transfer ability. ${ }^{6}$

A photothermal evaporation system is commonly composed of two components-the photothermal material and the supporting matrix. Various photothermal materials such as carbon materials, metallic nanoparticles, semiconductors, and conjugated polymers have been widely studied. ${ }^{7,8}$ They should have full-spectrum light absorbance and high light-to-heat conversion efficiency but their actual performance varies from each other, being dependent on the mechanism of photothermal conversion and light absorption capacity. ${ }^{9,10}$ Among them, carbon materials such as carbon nanotubes, ${ }^{11}$ graphene, ${ }^{12,13}$ carbon black, ${ }^{14}$ carbon aerogels as well as conjugated polymers such as polypyrrole (PPy) and polyaniline (PANI) ${ }^{15-17}$ have broadband absorption spectrum, high photothermal stability, and low density, making them ideal candidates. In addition, many carbon materials and conjugated polymers are of low cost and can be well incorporated into polymeric matrices to produce photothermal evaporation systems through various routes including in situ polymerization. ${ }^{18}$ Polymeric matrices 
not only provide support for photothermal materials but also undertake light, heat, and mass transfer in the evaporation systems. Therefore, an ideal polymeric matrix should have some substantial and structural requirements to eliminate the light loss from sunlight reflection and transmittance, disregard the heat loss into the environmental body, easily uptake the surrounding water, and deliver water rapidly inside the system. For example, the rough surface weakens light reflection and the thermal insulation eliminates heat loss, while the hydrophilicity and micro-channel facilitate the uptake and transport of water through capillary action. Therefore, porous and hydrophilic matrices have been extensively utilized in photothermal evaporation ${ }^{19,20}$ and self-floating devices have been achieved due to their low density. ${ }^{21-23}$

So far, photothermal evaporation performance has been improved significantly through the joint efforts of researchers. $^{23-27}$ Xiao et al. prepared an evaporation system that exhibits a double-layer cooperative effect with efficient light-absorption and water transportation, leading to a high evaporation rate of $1.37 \mathrm{~kg} \mathrm{~m} \mathrm{~m}^{-2} \mathrm{~h}^{-1}$ with a photothermal conversion efficiency of $88.7 \%$ under one-sun illumination. ${ }^{28}$ Chen at al. prepared a dual-functional material with a single component by a simply carbonizing biowaste platanus fruit, which can achieve a high evaporation rate up to $2.00 \mathrm{~kg} \mathrm{~m}^{-2}$ $\mathrm{h}^{-1}$ under one-sun irradiation by a 3D spherical evaporator. ${ }^{29}$ Ma et al. produced a device made from paulownia wood covalently bonded with MXene on the top, achieving an evaporation rate of $1.465 \mathrm{~kg} \mathrm{~m}^{-2} \mathrm{~h}^{-1}$ under one-sun irradiation with $96 \%$ conversion efficiency. ${ }^{30}$ In recent times, the photothermal desalination research is gradually concentrating on the structural modulation of photothermal systems, including the buildup of hierarchical porosity, ${ }^{31}$ the vertical alignment of micro-channels, ${ }^{32}$ the two-layer arrangement of the porous substrate, ${ }^{33,34}$ and the mushroom-like device. ${ }^{35}$ Since most of the current systems are of two components, photothermal materials should be uniformly dispersed insides the polymeric matrices to exert photothermal activity as efficiently as possible and the integration should be durable to avoid the escape of photothermal materials during the long-term application. However, little concerns have been devoted into this important issue. As for photothermal desalination, a large amount of salt solids accumulate, which clogs the porthole of water transport and weakens light absorption. Thus, Kou et al. developed lowcost and washable photothermal fabrics by dyeing traditional cotton fabrics with a carbon nanotube-based ink. ${ }^{36}$

Cryogel, a newly developed macroporous material, has been introduced as the matrix of photothermal evaporation systems. ${ }^{37}$ Compared with common (hydro)gels, cryogels are synthesized through the cryo-polymerization of monomers and cryo-crosslinkage of polymeric precursors at a temperature below the freezing point of the reaction mixture. They have interconnected macropores, high pore volume fraction, and sufficient mechanical strength. Also, they can be hydrophilic or hydrophobic, varying with the monomeric and polymeric precursors, while their porous morphology can be modulated through different routes. ${ }^{38}$ The most important is that cryogels take up the solvent very rapidly and hold the absorbed solvent firmly. Therefore, cryogels have been used as the matrix to incorporate various functional materials. ${ }^{39}$ In our previous research, ${ }^{40}$ polyacrylamide cryogel was introduced as the matrix for a mushroom-like photothermal evaporation device, whose evaporation rate and efficiency reached $1.63 \mathrm{~kg} \mathrm{~m}^{-2} \mathrm{~h}^{-1}$ and $89.2 \%$, respectively.

In this work, the poly(acrylic acid) (PAAc) cryogel was chosen as the matrix to incorporate polypyrrole (PPy) and polyaninile (PANI) since these conjugated polymers could be acid-doped by PAAc, facilitating their uniform distribution in the matrix. Also, graphene oxide was integrated during the preparation of the PAAc cryogel to testify the versatility of PAAc cryogel as the matrix, considering the possible polar interaction and hydrogen bonding. An evaporation system was designed to have the composite cryogel sheet on the top of the mushroom-shaped PAAc cryogel substrate, which was able to assemble or disassemble as desired. Due to the high sunlight absorbance of these photothermal materials along with sufficient water transport and heat insulation of the substrate, the evaporation system presented admirable performance of solar-to-heat water evaporation with the rate of pure water as high as $1.819 \mathrm{~kg} \mathrm{~m}^{-2} \mathrm{~h}^{-1}$ under one-sun irradiation and high durability even for salt water evaporation within 6 days.

\section{Experimental}

\subsection{Materials}

Graphite power was purchased from Beijing Jin-Long Technology Ltd. Graphene oxide (GO) was prepared from graphite power according to Hummers' method. ${ }^{41}$ Acrylic acid (AAc), pyrrole (Py), and aniline (ANI) were purchased from Aladdin as AR reagents and used after distillation. $\left(\mathrm{NH}_{4}\right)_{2} \mathrm{~S}_{2} \mathrm{O}_{8}$ (APS, AR), purchased from Sinophamn Chemical Reagent, was used after recrystallization. $N, N^{\prime}$-Methylenebisacrylamide (MBAm), L-ascorbic acid ( $\mathrm{Vc}), \mathrm{FeCl}_{3}$, and other chemicals of AR grade were purchased from Sinopharm Chemical Reagent and used as received. Distilled water was used in all the experiments.

\subsection{Synthesis of PAAc cryogels and hydrogels without photothermal materials}

PAAc cryogel was synthesized according to literature ${ }^{42}$ with modification. AAc $(20 \mathrm{mmol}), \mathrm{MBAm}(1 \mathrm{mmol})$, and $\mathrm{Vc}$ $(0.24 \mathrm{mmol})$ were dissolved in distilled water $(9 \mathrm{~mL})$, while APS aqueous solution $\left(0.2 \mathrm{mmol} \mathrm{mL} \mathrm{mL}^{-1}, 1.0 \mathrm{~mL}\right)$ was prepared separately. After both aqueous solutions were pre-cooled in an ice bath for $10 \mathrm{~min}$, they were mixed homogeneously. Then, the mixture was instantly transferred into a Petri dish with $6 \mathrm{~cm}$ diameter and kept at $-18{ }^{\circ} \mathrm{C}$ for $24 \mathrm{~h}$ to complete the cryopolymerization. The obtained PAAc cryogel was immersed in distilled water for $48 \mathrm{~h}$ with exchanging distilled water every $6 \mathrm{~h}$ to remove the unreacted monomer and un-crosslinked polymer. Afterwards, the dried cryogel sheet was obtained through lyophilization at $-44{ }^{\circ} \mathrm{C}$ for $48 \mathrm{~h}$. Based on the remaining mass of the cryogel sheet, the monomer conversion and gel fraction were estimated. 
The preparation of the PAAc cryogel substrate was carried out through the same procedure as that above in a mushroom-shaped mould (Fig. S1a, ESI $\dagger$ ). A cryogel sheet and a mushroom-shaped cryogel were assembled within polystyrene (PSt) foam (Fig. S1b, ESI $\dagger$ ), resulting in the photothermal evaporation system.

With the same polymerization procedure and vessels, the PAAc hydrogel substrate was prepared through common polymerization at room temperature.

\subsection{Synthesis of the PAAc composite cryogels and hydrogels with photothermal materials}

As for the preparation of the PAAc composite cryogels with PPy or PANI (PAAc-PPy or PAAc-PANI cryogel), a dried sheet of PAAc cryogel ( $6 \mathrm{~cm}$ in diameter) was kept in $1 \mathrm{M} \mathrm{HCl}$ solution $(140 \mathrm{~mL})$ containing aniline or pyrrole $(0.25 \mathrm{~g})$ at $0{ }^{\circ} \mathrm{C}$ for $60 \mathrm{~min}$ for monomer absorption. Afterwards, APS $(0.785 \mathrm{~g}$ for aniline) or $\mathrm{FeCl}_{3}(3.0 \mathrm{~g}$ for pyrrole) in $\mathrm{HCl}$ solution $(10 \mathrm{~mL})$ was added under magnetic stirring. The mixture of oxidative coupling polymerization was stood at $5{ }^{\circ} \mathrm{C}$ for $6 \mathrm{~h}$. Finally, the composite cryogels were washed with ethanol/water mixture ( $50: 50$ by volume) several times and lyophilized.

The preparation of the composite cryogel with graphene oxide (PAAc-GO cryogel) was quite similar to that of pure PAAc cryogel, except that AAc and MBAm were dissolved beforehand in $\mathrm{GO}$ aqueous dispersion $\left(2 \mathrm{mg} \mathrm{mL}^{-1}, 9 \mathrm{~mL}\right.$ ). The obtained PAAc-GO cryogel was kept in a Vc solution $\left(3 \mathrm{mg} \mathrm{L}^{-1}, 100 \mathrm{~mL}\right)$ at $60{ }^{\circ} \mathrm{C}$ for $12 \mathrm{~h}$ to convert graphene oxide into reduced GO (rGO). ${ }^{4,44}$ Then, the PAAc-rGO cryogel was washed with distilled water and lyophilized. The color change from brown to black indicated the reduction of GO (Fig. S2, ESI $\dagger$ ).

\subsection{Swelling behavior and porosity fraction of the cryogels/ hydrogels}

A dried cryogel sheet was soaked in $100 \mathrm{~mL}$ distilled water. At regular intervals, the sample was taken out, the surface water was removed with filter paper, and the swollen cryogel was weighed. The above step was repeated until the mass no longer changed. The swelling degree ( $\mathrm{SD}, \mathrm{g} \mathrm{g}^{-1}$ ) was obtained according to eqn (1).

$$
\mathrm{SD}=\frac{m_{\mathrm{s}}-m_{0}}{m_{0}}
$$

where $m_{\mathrm{s}}$ and $m_{0}$ is the mass of the swollen and the dried cryogel, respectively.

The apparent density $(\rho)$ of the dried cryogel was determined by its mass and dimensional size. The diameter and thickness of the cryogel sheet are shown in Fig. S1c and d (ESI $\dagger)$. The actual density $\left(\rho_{0}\right)$ of the cryogel substance excluding the pores was calculated according to eqn (2). The related density values are collected in Table S1 (ESI $\dagger)$. Thus, the porosity ( $\phi$, vol\%) of the cryogel was determined according to eqn (3).

$$
\begin{gathered}
\rho_{0}=\rho_{0, \mathrm{PAAc}} \times w_{\mathrm{PAAc}}+\rho_{0, \mathrm{tm}}+w_{\mathrm{tm}} \\
\varnothing=\left(1-\frac{\rho}{\rho_{0}}\right) \times 100
\end{gathered}
$$

where $w_{\text {PAAc }}$ and $w_{\text {tm }}$ is the mass fraction of PAAc and the photothermal material in the composite cryogel, respectively. The parameter of $\rho_{0, \text { PAAc }}$ and $\rho_{0, \text { tm }}$ represents the density of pure PAAc and the photothermal material, respectively. The density value is $1.200,0.967,1.022$, and $2.100 \mathrm{~g} \mathrm{~cm}^{-3}$ for PAAc, PPy, PANI, and graphene, respectively.

\subsection{Photothermal evaporation with different cryogels}

The measurement setup of photothermal evaporation is demonstrated in Scheme S1 (ESI $\dagger$ ). The experiment was performed at room temperature $\left(25^{\circ} \mathrm{C}\right)$ and humidity (45-55\%) using a solar simulator (CEL-HXF300/CEL-HXUV 300) equipped with an AM 1.5G filter and the incident light was adjusted to $1 \mathrm{~kW} \mathrm{~m}{ }^{-2}$ (one-sun irradiation) with an optical power meter (PL-MW2000). In each test, after a mushroom-shaped PAAc cryogel was inserted through the PSt foam button, a composite cryogel sheet (cut to the diameter of $3.0 \mathrm{~cm}$ ) was kept in touch with the top of the mushroom-shaped cryogel and entrapped inside the PSt foam. Then, the integrated system was placed across a beaker filled with water, with the tip of the mushroomshaped cryogel contacting the water (Fig. S1b, ESI $\dagger$ ). The temperature of the evaporation system was recorded with an infrared camera (Fluke TiS40). The mass loss was measured on a high accuracy balance (FA2204, $0.1 \mathrm{mg}$ in accuracy) and a computer installed with a software (ShuDaXia) was connected to the balance to record the mass data in real time.

\subsection{Characterization}

The chemical structure of the cryogel was analyzed by Fourier transform infrared spectroscopy (FT-IR, Nicolet 6700, Thermo Fisher, USA) in $\mathrm{KBr}$ pellets IN the wavenumber range of 4000$500 \mathrm{~cm}^{-1}$, scanning step of $4 \mathrm{~cm}^{-1}$, and 16 scanning runs. The Raman spectra of PAAc-GO/rGO cryogels were collected by a laser confocal scanning Raman microscope (LABRAM-HR, France) with a laser source of $532 \mathrm{~nm}$. Thermogravimetric analysis (TGA, Q5000IR, USA) was carried out from 25 to $800{ }^{\circ} \mathrm{C}$ at a heating rate $10{ }^{\circ} \mathrm{C} \mathrm{min}^{-1}$. Solar reflectance and transmittance measurement from 250 to $2500 \mathrm{~nm}$ were performed using a UV-vis-NIR spectrophotometer (SOLID3700, USA) equipped with an integrating sphere, where the absorption efficiency $(A)$ was calculated by $A=(1-R-T)(R$ and $T$ is the reflectance and transmission efficiency, respectively). Tungsten filament scanning electron microscopy (TFSEM, EVO18, Germany) was used to study the morphology of the dried cryogel and the hydrogel. The porosity, channel size, and pore area of the cryogel were evaluated with a mercury intrusion porosimeter (AutoPore IV 9500 2.03.01, USA). To confirm the distribution of PANI and PPy in the PAAc cryogel, energy dispersive X-ray spectroscopy (EDX, GeminiSEM 500, Germany) coupled to SEM was used. Thermal diffusion coefficiency was determined by a laser thermal conductivity meter (LFA-467, Germany). Specific heat capacity was characterized through differential scanning calorimetry (DSC, Q2000, USA). The contents of metal ions in the collected water after photothermal evaporation were measured by inductively coupled plasmamass spectrometry (ICP-MS PGS-2, Zeiss, USA). 


\section{Results and discussion}

\subsection{Preparation of different cryogels}

Pure PAAc cryogels were prepared through redox-initiated cryopolymerization with MBAm as the crosslinking monomer at $-18{ }^{\circ} \mathrm{C}$ (Scheme 1). Based on the mass of the obtained cryogel, monomer conversion was nearly complete and the gel fraction exceeded 95\%. FTIR, Raman, and TGA results confirmed the chemical composition of all the cryogels, as shown in Fig. S3 and S4 (ESI $\dagger$ ). The carboxylic group of PAAc has not only strong acid-base interaction with the Py and ANI units in the monomer and polymer but also sufficient hydrogen bonding with GO having oxygen-containing groups, which facilitated the uniform distribution and tight integration of photothermal materials inside the PAAc cryogel.

\subsection{Porous morphology of the cryogels and the distribution of the photothermal component}

The real density of individual components in the composite cryogel $\left(\rho_{0}\right)$ and the apparent density of the cryogel/hydrogel $\left(\rho_{\text {app }}\right)$ are listed in Table S1 (ESI $\left.\dagger\right)$. According to eqn (3), the porosity in volume fraction $(\phi)$ is obtained and also summarized in Table S1 (ESI $\dagger$ ). All the cryogels have low apparent density $\left(0.136-0.171 \mathrm{~g} \mathrm{~cm}^{-3}\right.$ ) and high porosity (86-89 vol\%), which effectively helps water transport and lessens heat loss. On the contrary, the PAAc hydrogel has higher density $(0.360 \mathrm{~g}$ $\mathrm{cm}^{-3}$ ) and lower porosity fraction (70 vol\%), which limits rapid water transport inside the PAAc hydrogel. As shown in Fig. S5 (ESI $\dagger$ ), the porosity of the cryogel and hydrogel tested by the mercury porosimeter is consistent with the results obtained by density calculation. The total area per unit mass of the cryogel is more than ten times that of the hydrogel.

TFSEM was used to observe the pore morphology of different cryogels with regard to the PAAc hydrogel and their images are shown in Fig. 1. From Fig. 1a, open macro-pores are not significantly observed for the PAAc hydrogel and some bulblike pores are present in the inset with high magnification. As suggested by Fig. $1 \mathrm{~b}-\mathrm{f}$, all the cryogels contrarily exhibit the macroporous morphology and the pores are open and interconnected, which is important for easy water transport inside

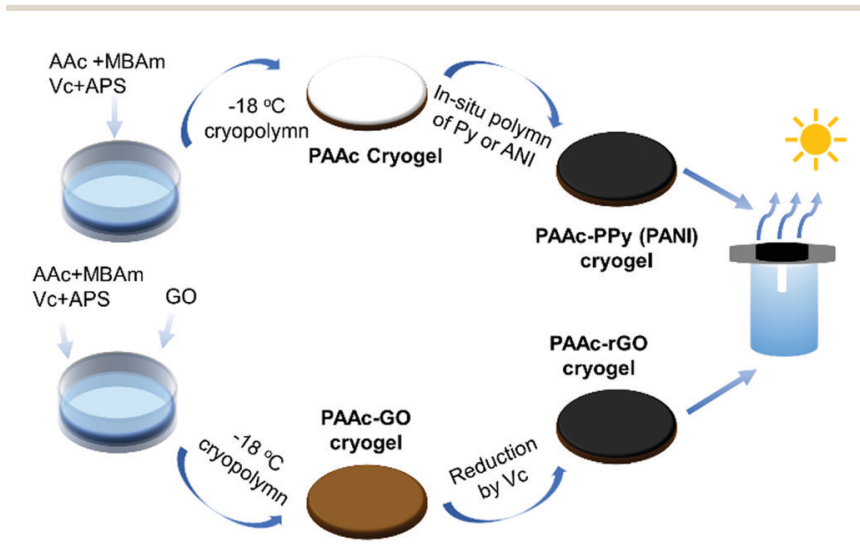

Scheme 1 Preparation procedure for PAAc-PPy (PANI) or PAAc-GO ( $r G O)$ cryogel and the assembly of the photothermal evaporation system.

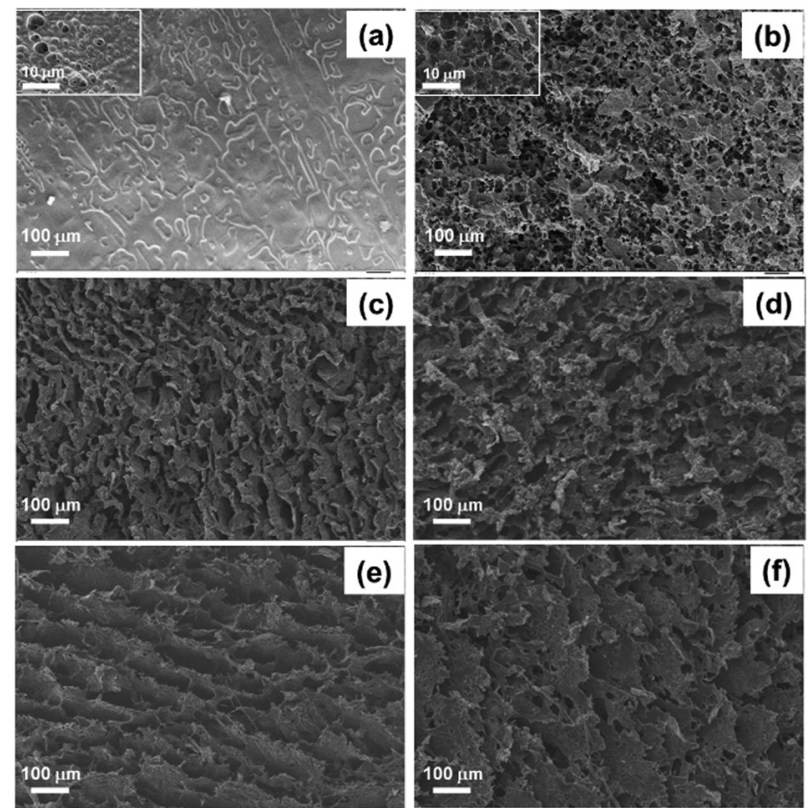

Fig. 1 TFSEM images of the PAAc hydrogel (a) and the cryogels of PAAC (b), PAAC-PANI (c), PAAc-PPy (d), PAAc-GO (e), and PAAc-rGO (f).

the cryogels. As for the pure PAAc cryogel, the macropores are more and the pore-wall is thin. After the polymerization of ANI or Py inside the PAAc cryogel, the pore-wall of both the PAAcANI and PAAc-PPy cryogels becomes much thicker and the macropores seem to reduce, as seen in Fig. 1c and d, suggesting that the formed PANI and PPy are tightly and uniformly attached onto the pore-wall. SEM coupled with EDX was used to further confirm the uniform distribution of PANI and PPy inside the cryogels, as shown in Fig. S6 and S7 (ESI + ), the element mapping images of the PAAc-ANI and PAAc-PPy cryogels, respectively. The images of $\mathrm{C}, \mathrm{O}$, and $\mathrm{N}$ elements reveal the same pattern from vertical and lateral viewing.

As indicated in Fig. 1e and f, the PAAc-GO and PAAc-rGO cryogels display lamellar-like macroporous morphology, induced by the presence of graphene nano-sheets. Compared to other cryogels, their macropores are much larger and the pore-walls are loosely piled up.

The uniform distribution of photothermal materials inside the cryogels is indirectly supported by FTIR and TGA analysis. As indicated by the local FTIR spectra (Fig. S8, ESI $\dagger$ ), the signal of $\mathrm{C}=\mathrm{O}$ stretching vibration for the composite cryogels displays a red-shift to a different extent, which follows the sequence PAAc-PPy $>$ PAAC-PANI $>$ PAAc-GO $>$ PAAc-rGO. This red-shift is caused by hydrogen bonding between the carboxylic group of PAAc and the polar groups of the photothermal components. Also, the signal of $\mathrm{C}-\mathrm{O}$ stretching vibration of $\mathrm{COOH}$ identifies the above interaction. This signal is present at $\sim 1252 \mathrm{~cm}^{-1}$ in the FTIR spectra of PAAc, PAAc-GO, and PAAc-rGO cryogels, with distinguishable red-shift for the PAAc-PANI cryogel $\left(1242 \mathrm{~cm}^{-1}\right.$ ) due to acidic doping. This signal is absent for the PAAc-PPy cryogel, suggesting that the acid doping of PPy by PAAc is much extensive. Such a strong interaction also causes a 
difference in the TGA thermogram between the PAAc-PPy cryogel and others (Fig. S4b, ESI $\dagger$ ).

\subsection{Light absorption, thermal insulation, and water uptake of different cryogels}

While the $\pi$-conjugated structure of the photothermal materials facilitates sunlight absorbance over a widespread spectrum, ${ }^{10}$ the photothermal systems should eliminate the light transmittance and reflection. Thus, UV-vis-NIR reflectance and transmittance spectroscopy was used to evaluate sunlight absorbance, as demonstrated in Fig. 2 .

The transmittance and reflectance of the PAAc cryogel in the wavelength range of $300-1400 \mathrm{~nm}$ is very high up to 17 and $76 \%$, respectively. As shown in Fig. 2a, after introducing photothermal materials, the transmittance of the PAAc-GO cryogel drops below $4 \%$ and others are nearly zero in the whole spectrum. Meanwhile, the reflectance of the PAAc composite cryogels (Fig. 2b) decreases significantly compared with the PAAc cryogel. The reflectance decrease for the PAAc-GO cryogel is the weakest with the reflectance above $20 \%$ due to its lamellar morphology. After GO is reduced, the reflectance of the PAAc-rGO cryogel falls to about $17 \%$ in the visible/nearinfrared region. Both the PAAc-PPy and PAAc-PANI cryogels have reflectance less than $5 \%$. Therefore, compared with sunlight absorption by the PAAc cryogel (less than $60 \%$, especially as low as $8 \%$ in the visible region) in Fig. 2c, composite cryogels with photothermal materials show a significant increase in sunlight absorption, especially for the PAAc-PPy and PAAcPANI cryogels. It should be mentioned that the sunlight absorbance of the PAAc-PPy cryogel is almost $100 \%$ in the whole spectrum and the light intensity in the visible region is much higher as indicated by the solar spectral radiance. Based on the intensity distribution of sunlight over wavelength, the overall efficiency of sunlight absorption $\left(\eta_{\text {abs }}\right)$ by different cryogels are calculated in the range of 200-2000 $\mathrm{nm}$ according to eqn (4)

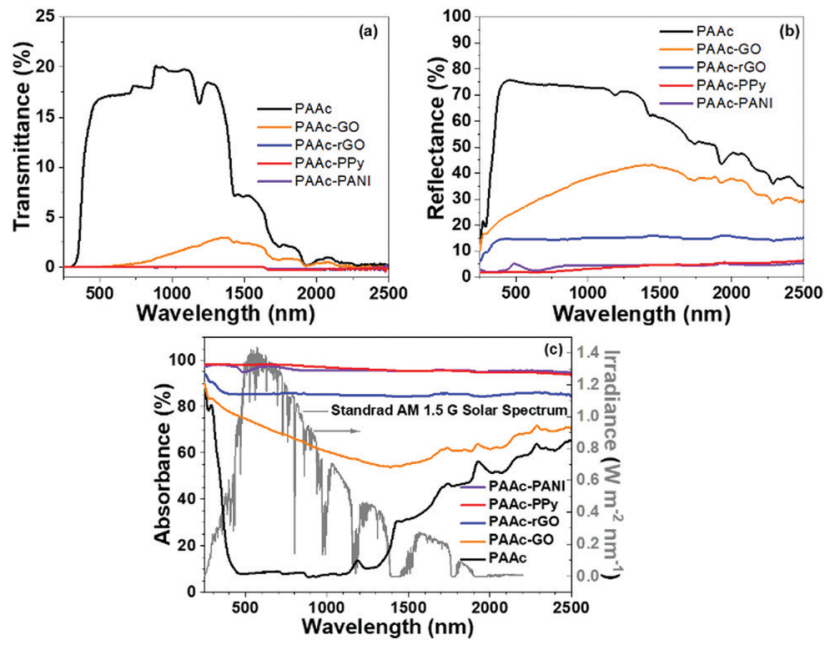

Fig. 2 UV-vis-NIR transmittance (a), reflectance (b), and absorbance (c, left $Y$-axis) spectra of different cryogels, and solar spectral radiance (c, right $Y$-axis). and the results are listed in Table $\mathrm{S} 2(\mathrm{ESI} \dagger)$.

$$
\eta_{\mathrm{abs}}=\sum_{200}^{2000} \eta_{\lambda} I_{\lambda} / \sum_{200}^{2000} I_{\lambda}
$$

where $\eta_{\lambda}$ and $I_{\lambda}$ is the absorbance and intensity at each wavelength $(\lambda)$, respectively.

The sunlight absorption absolutely increases the cryogel temperature due to solar-to-heat conversion. Fig. S9 (ESI $\dagger$ ) shows the IR camera images of different dry cryogels after one-sun irradiation for $20 \mathrm{~min}$. The maximum temperature inside the light spot $\left(T_{\max }\right)$ is as high as about 86.7 and $89.1{ }^{\circ} \mathrm{C}$ for the PAAc-PPy and PAAc-PANI cryogels. The maximum temperature of dry PAAc-GO and PAAc-rGO reaches 78.2 and $76.1{ }^{\circ} \mathrm{C}$, respectively. However, the maximum temperature of the pure PAAc cryogel is much lower $\left(35.3{ }^{\circ} \mathrm{C}\right)$, reflecting that photothermal materials prompt sunlight absorption. Fig. 3 offers the temperature outlook during the steady photothermal evaporation of pure water in different cases after one-sun irradiation for $20 \mathrm{~min}$. Since there exists temperature inhomogeneity over the cryogel surface under irradiation, as indicated in Fig. 3, the average temperature over the cryogel sheet $\left(T_{\text {ave }}\right)$ under one-sun irradiation has been calculated. The temperature difference between the cryogel sheet and water reservoir is taken as $\left(T_{\text {ave }}-T_{\text {room }}\right)$. All the temperature data are summarized in Table S2 (ESI $\dagger$ ).

Without any cryogel, the surface temperature of pure water can reach only $30.9{ }^{\circ} \mathrm{C}$ (Fig. 3a), while that of the PAAc cryogel sheet can get to $35{ }^{\circ} \mathrm{C}$ (Fig. 3b), which is close to that of the dried PAAc cryogel. On the other hand, with the incorporation of photothermal materials, the surface temperature of the
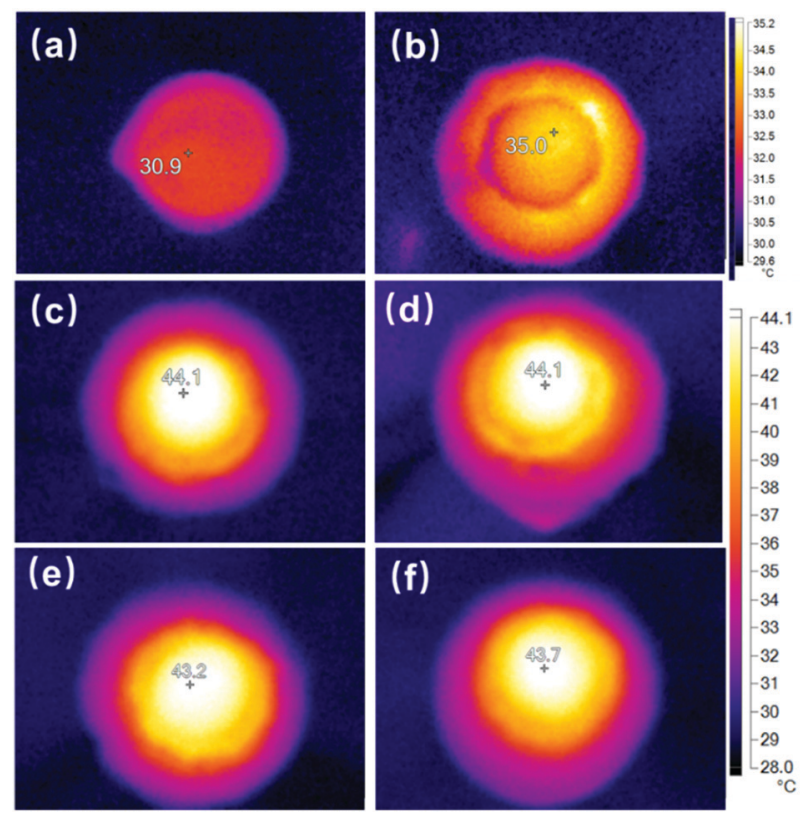

Fig. 3 IR camera images of pure water and the cryogel surface during photothermal evaporation of pure water after one-sun irradiation for 20 min (a: pure water, b: PAAc, c: PAAc-PPy, d: PAAc-PANI, e: PAAcGO, f: PAAc-rGO). 
cryogel sheet during photothermal evaporation exceeds $43{ }^{\circ} \mathrm{C}$ for all the composite cryogels and the elevated temperatures are much lower than those for dried cryogels, confirming that the light energy absorbed by photothermal materials is mostly consumed for water evaporation. It should be noted that the surface temperature of the photothermal system has two opposite influences on water evaporation. One is the less evaporation enthalpy of water at higher temperature, which prompts water evaporation. Another is the less heat loss from cryogels to the environment at lower temperature.

A photothermal evaporation system should require low thermal conductivity to prevent heat transfer to the surrounding environment and water reservoir. The thermal conductivity was determined to be 0.177, 0.056, 0.049, 0.043, and $0.038 \mathrm{~W} \mathrm{~m}^{-1} \mathrm{~K}^{-1}$ for the PAAc-PPy, PAAc-PANI, PAAc-GO, PAAc-rGO, and PAAc cryogels, respectively. These values are much lower than that of water $\left(0.592 \mathrm{~W} \mathrm{~m}^{-1} \mathrm{~K}^{-1}\right.$ at standard state) and comparable to that of the PS foam $\left(\sim 0.043 \mathrm{~W} \mathrm{~m}^{-1} \mathrm{~K}^{-1}\right)$. The excellent thermal insulation of all the cryogels can effectively minimize heat loss. Considering a little higher thermal conductivity of the PAAc-PPy cryogel, all the composite cryogel sheets are mounted on the mushroom-shaped PAAc cryogel substrate to further eliminate heat transfer and maintain the same evaporation conditions.

Water uptake by cryogels and water transport inside the cryogels are also important to achieve excellent photothermal evaporation. Thus, the water absorption behaviors of the PAAc hydrogel and different cryogels were compared through swelling kinetics study, as shown in Fig. 4a.

It takes about $4 \mathrm{~h}$ for the PAAc hydrogel (H-PAAc) to reach swelling equilibrium and the swelling ratio is about $4.6 \mathrm{~g} \mathrm{~g}^{-1}$. On the contrary, all the cryogels reach swelling equilibrium within $10 \mathrm{~s}$ and the swelling ratio of the PAAc cryogel (C-PAAc) is about $7.3 \mathrm{~g} \mathrm{~g}^{-1}$, as shown in Fig. $4 \mathrm{~b}$. To verify the capillary action, a rectangular sheet of the PAAc cryogel or hydrogel $(1 \mathrm{~cm} \times 6 \mathrm{~cm})$ was used to absorb a thinned solution of red ink, as recorded in Video S1 and S2 (ESI $\dagger$ ), respectively. It is seen that the cryogel transfers red ink to the uppermost end in only $25 \mathrm{~s}$ while the hydrogel transfers only $0.5 \mathrm{~cm}$ after $120 \mathrm{~s}$. The rapid water uptake of the cryogels and the speedy transport of water inside the cryogels is attributed to their interconnected and hierarchical macroporosity. Later, a comparison of the photothermal evaporation of pure water between the PAAc


Fig. 4 Swelling kinetics of the PAAc-hydrogel (a) and different cryogels (b). hydrogel and cryogel as the substrates provides additional support.

\subsection{Photothermal evaporation of pure water with different cryogels and energy consideration}

To evaluate the photothermal evaporation performance of cryogels, a mushroom-shaped PAAc cryogel was used as a pedestal covered with one composite cryogel sheet. Although the apparent density of each cryogel is lower than water, the completely swollen cryogels are still unable to self-float on water. In addition, elevated temperature from sunlight absorption might bring out the heat loss. Therefore, all the tested cryogels are entrapped in the PSt foam with the mushroom tip keeping in touch with the water reservoir (Scheme S1 and Fig. S1b, ESI $\dagger$ ).

The steady photothermal evaporation of pure water without and with the cryogels is shown in Fig. 5a. In the dark, the evaporation rate of pure water is $0.350 \mathrm{~kg} \mathrm{~m}^{-2} \mathrm{~h}^{-1}$. Under onesun irradiation, the evaporation rate of pure water without and with the pure PAAc cryogel is almost the same (0.553 and 0.555 $\mathrm{kg} \mathrm{m} \mathrm{m}^{-2} \mathrm{~h}^{-1}$, respectively). This result proposes that the PAAc cryogel does not affect the water transport and evaporation. After a composite cryogel sheet is used, the evaporation rate increases significantly by more than three folds. The PAAc-PPy cryogel has the highest rate $\left(1.819 \mathrm{~kg} \mathrm{~m}^{-2} \mathrm{~h}^{-1}\right)$, the PAAc-PANI cryogel stands next $\left(1.678 \mathrm{~kg} \mathrm{~m}^{-2} \mathrm{~h}^{-1}\right)$, while the PAAc-rGO and PAAc-GO cryogels have a similar evaporation rate, which were 1.585 and $1.551 \mathrm{~kg} \mathrm{~m}^{-2} \mathrm{~h}^{-1}$, respectively.

During steady photothermal evaporation, the incident sunlight is absorbed by the composite cryogel sheet and the absorbed solar energy is converted into heat to accelerate water evaporation. According to the UV-vis-NIR absorbance spectrum (Fig. 2c), both the PPAc-PPy and PAAc-PANI cryogels have almost $100 \%$ absorption of incident light but the absorption percent of both the PAAc-GO and PAAc-rGO cryogels is relatively low. Thus, the high evaporation rate for the PAAc-PPy and PAAcPANI cryogels could be assigned to their efficient ability of sunlight absorption. Considering that PPAc-PPy and PAAc-PANI cryogels have similar sunlight absorption efficiency but different evaporation rate and that the PAAc-GO cryogel has lower light absorption efficiency than the PAAc-rGO cryogel but their evaporation rates are fairly close, there are other factors influencing the photothermal evaporation rate.
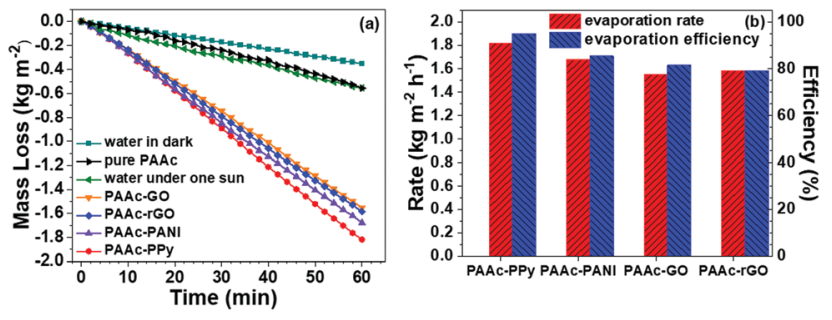

Fig. 5 (a) Cumulative mass loss of water versus time for the photothermal evaporation of pure water in dark and under one-sun irradiation with/ without different cryogels; (b) evaporation rate and efficiency of different cryogels. 
Within a certain period, the absorbed sunlight energy $\left(I_{\text {abs }}\right)$ during steady photothermal evaporation is consumed by waterto-vapor conversion $\left(Q_{\text {evp }}=R_{\text {real }} \times \Delta H_{\text {evap }}\right)$, heating evaporated water from room temperature to the temperature of the cryogel sheet $\left(Q_{\text {heating,water }}=C_{\text {water }} \times \Delta T\right)$ and possible heat loss $\left(Q_{\text {loss }}\right)$, as indicated by the following equations based on the energy balance.

$$
\begin{gathered}
\eta_{\mathrm{abs}} I_{\mathrm{inc}}=I_{\mathrm{abs}}=R_{\text {real }} \times\left(\Delta H_{\text {evap }}+C_{\text {water }} \times \Delta T\right)+Q_{\text {loss }} \\
R_{\text {real }}=R_{\text {light }}-R_{\text {dark }}
\end{gathered}
$$

where $R_{\text {real }}$ is the real light-driven evaporation rate, which is equal to the evaporation rate under light irradiation $\left(R_{\text {light }}\right)$ subtracted by the evaporation rate in dark $\left(R_{\text {dark }}\right) . I_{\text {inc }}$ is the incident light intensity (one-sun as $1 \mathrm{~kW} \mathrm{~m}^{-2}$ ). $\eta_{\text {abs }}$ is the light absorbance, i.e., the light absorption efficiency. $C_{\text {water }}$ is the specific heat capacity of water and is taken as a constant of $4.18 \mathrm{~J} \mathrm{~g}^{-1} \mathrm{~K}^{-1} . \Delta T$ is the temperature increment of water during the photothermal evaporation and taken as the temperature difference between the cryogel surface under irradiation and the water reservoir. $\Delta \mathrm{H}_{\text {evap }}$ is the evaporation enthalpy of water, which decreases with the increase in temperature. $\Delta \mathrm{H}_{\text {evap }}$ is about 2250 and $2400 \mathrm{~J} \mathrm{~g}^{-1}$ at 100 and $40{ }^{\circ} \mathrm{C}$ under standard pressure, respectively. Since $\Delta T$ is about $15^{\circ} \mathrm{C}$ and $C_{\text {water }} \times \Delta T$ is about $3 \%$ of $\Delta \mathrm{H}_{\text {evap }}$ (Table S3, ESI $\dagger$ ), lessening $\Delta \mathrm{H}_{\text {evap }}$ is an important and efficient approach to increase the evaporation rate. As mentioned above, the decrease in $\Delta \mathrm{H}_{\text {evap }}$ can be achieved with the increase in the evaporation temperature. In addition, considering that $\Delta \mathrm{H}_{\text {evap }}$ is attributed to the breakdown of intermolecular interaction among water molecules, especially hydrogen bonding, the appropriate interaction between water and the cryogel sheet could prompt the decrease of $\Delta \mathrm{H}_{\text {evap }}$. This assumption is experimentally supported with the phenomenon that PAAc-GO with oxygen-containing groups exhibits an apparent evaporation efficiency ( $\left.\eta_{\text {evap,app }}\right)$ of $123 \%$ (Table S3, ESI $\dagger$ ), considering no influence of the cryogel structure on $\Delta H_{\text {evap }}$.

As proposed by the literature, ${ }^{24,45}$ there are three states of water molecules in the composite cryogel, namely, free water, transitional water, and bound water. Molecules of free water are connected with each other through hydrogen bonds and confined in dynamic and stable clusters, making water evaporation more difficult. Since there are still hydrogen bonds and other interactions between water molecules and the cryogel porewall, the molecules of bound water are also confined and difficult to evaporate. As for transitional water, water molecules are loosely confined and more likely to escape from the water cluster and the cryogel network. Therefore, the matrix as well as the photothermal material should be delicately chosen to balance the interactions of water-to-water and water-to-matrix, which could lessen the real evaporation enthalpy of water.

Because of the very low thermal conductivity of cryogels and the entrapping of the cryogel sheet inside the PSt foam, the heat loss $\left(Q_{\text {loss }}\right)$ in eqn (5) is neglected. Thus, $\eta_{\text {evap }}$ and the total evaporation efficiency $\left(\eta_{\text {total }}\right)$ are calculated according to the following equations.

$$
\begin{aligned}
& \eta_{\text {evap }}=R_{\text {real }} \times \frac{\left(\Delta H_{\text {evap }}+C_{\text {water }} \times \Delta T\right)}{I_{\text {abs }}} \\
& \eta_{\text {total }}=R_{\text {real }} \times \frac{\left(\Delta H_{\text {evap }}+C_{\text {water }} \times \Delta T\right)}{I_{\text {inc }}}
\end{aligned}
$$

Taking $\Delta H_{\text {evap }}$ as $2400 \mathrm{~J} \mathrm{~g}^{-1}\left(40{ }^{\circ} \mathrm{C}\right)$, the total evaporation efficiency for different composite cryogels is more than $82 \%$, which is comparable with the literature reports (Table S4, ESI $\dagger$ ). However, it is questionable that the total evaporation efficiency for the PAAc-PPy cryogel is nearly $100 \%$, considering the possible heat loss and the deviation of $\Delta H_{\text {evap }}$ from its real value at temperature the than $40{ }^{\circ} \mathrm{C}$. Although $\Delta H_{\text {evap }}$ for photothermal evaporation has been measured with differential scanning calorimetry (DSC) in previous reports, ${ }^{24}$ those obtained values do not reflect the real evaporation enthalpy at the operation temperature due to the wide temperature scan of DSC.

\subsection{Photothermal evaporation of pure water and water transport consideration}

As discussed beforehand, PAAc cryogels have excellent ability to transport water due to their high hydrophilicity and hierarchical porosity. Excellent water transport ensures instant supply from the water reservoir to cover the evaporated water in the composite cryogel sheet, especially in the case of high evaporation rate. Therefore, photothermal evaporation under four-sun irradiation has also been performed with composite cryogel sheets and mushroom-shaped PAAc substrates. Upon light irradiation, water mass loss was immediately recorded. Fig. 6 shows the variation in the mass loss versus time within $1 \mathrm{~h}$. In the first $10 \mathrm{~min}$, all the cases exhibit a slower mass loss because the absorbed light energy is partially consumed to heat the cryogel sheet. After $20 \mathrm{~min}$, photothermal evaporation goes steady for all the cryogel sheets and the evaporation rate within 20-60 min was determined to be 6.492, 5.663, 5.652, and 5.407 $\mathrm{kg} \mathrm{m}^{-2} \mathrm{~h}^{-1}$ for the PAAc-PPy, PAAc-PANI, PAAc-rGO, and PAAcGO cryogel sheets, respectively. With mushroom-shaped PAAc cryogel as the substrate, water transport inside is sufficiently high to satisfy surficial water evaporation. After the replacement of the substrate with the PAAc hydrogel, the water mass loss behaviors differ, as suggested by the hollow data points of
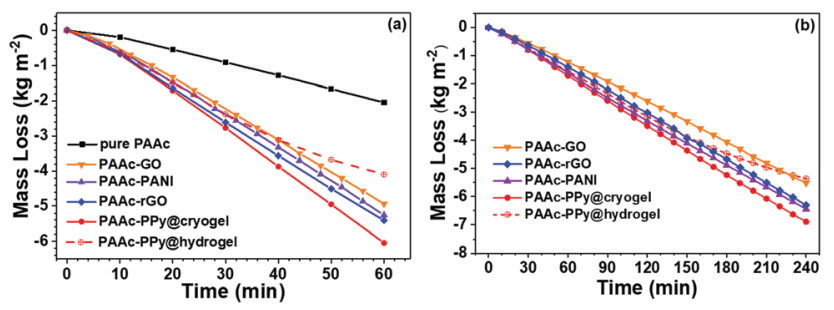

Fig. 6 (a) Photothermal evaporation of pure water under four-sun irradiation; (b) long-term photothermal evaporation of pure water under onesun irradiation (solid point: PAAc cryogel as the substrate, hollow point: PAAc hydrogel as the substrate). 
the PAAc-PPy@hydrogel. From $40 \mathrm{~min}$, the fitting line slopes up, indicating the decrease in the evaporation rate. Based on the slopes within $20-40 \mathrm{~min}$ and $40-60 \mathrm{~min}$, the average evaporation rate in the related duration is 4.871 and $2.962 \mathrm{~kg}$ $\mathrm{m}^{-2} \mathrm{~h}^{-1}$, respectively. This result is caused by the slow water transport inside the PAAc hydrogel, leading to the shortage of water in the PAAc-PPy cryogel sheet. It can be observed that the PAAc-PPy cryogel sheet starts to distort at $40 \mathrm{~min}$ and finally becomes warped, as shown in Fig. S10a (ESI $\dagger$ ). The surplus heat has to increase the temperature of the PAAc-PPy cryogel sheet. After being irradiated with four-sun light for $1 \mathrm{~h}, T_{\text {ave }}$ and $T_{\max }$ for the PAAc-PPy@cryogel remains 57.0 and $60.1{ }^{\circ} \mathrm{C}$, respectively. However, due to insufficient water supply when the hydrogel is used as the substrate, the light-to-vapor conversion cannot happen smoothly and the light energy absorbed by the photothermal layer is mostly converted into heat. $T_{\text {ave }}$ and $T_{\max }$ for the PAAc-PPy@hydrogel increases to 150.0 and $174.7{ }^{\circ} \mathrm{C}$, respectively. All the data are shown in Table S5 (ESI $\dagger$ ).

Long-term photothermal evaporation has been carried out under one-sun irradiation for $4 \mathrm{~h}$. The mushroom-shaped PAAc cryogel and the composite cryogel sheet were assembled and the starting point was taken as that upon light irradiation. The results are demonstrated as solid points in Fig. 6b. Over a duration of $4 \mathrm{~h}$, the fitting lines seems linear for the PAAc cryogel, indicating the constant evaporation rate and sufficient water transport inside the PAAc cryogel. Also, the PAAc-PPy cryogel sheet has the highest evaporation rate and PAAc-GO the lowest. The situation is different when the mushroom-shaped PAAc hydrogel instead of the PAAc cryogel is used as the substrate. As shown with hollow points with dotted line for the PAAc-PPy@hydrogel, the evaporation rate begins to significantly decrease at $150 \mathrm{~min}$. During the first half an hour (0-30 min), the evaporation rate for obviously PAAc-PPy@hydrogel $\left(1.483 \mathrm{~kg} \mathrm{~m}^{-2} \mathrm{~h}^{-1}\right)$ is comparable with that for obviously PAAcPPy@cryogel (1.587 $\left.\mathrm{kg} \mathrm{m}^{-2} \mathrm{~h}^{-1}\right)$. During the second half an hour (30-60 $\mathrm{min})$, the evaporation rate for both the cases is a little different $\left(1.627 \mathrm{~kg} \mathrm{~m} \mathrm{~m}^{-2} \mathrm{~h}^{-1}\right.$ for obviously hydrogel $v s$. $1.833 \mathrm{~kg} \mathrm{~m}^{-2} \mathrm{~h}^{-1}$ for the cryogel). The large deviation occurs within $150-180 \mathrm{~min}$, where the evaporation rate is 1.748 and $1.186 \mathrm{~kg} \mathrm{~m}^{-2} \mathrm{~h}^{-1}$ for the PAAc-PPy@cryogel and PPy@hydrogel, respectively. The continuous decline of the evaporation rate is caused by the low water transport inside the PAAc hydrogel substrate. As shown in Fig. S10b (ESI $\dagger$ ), the PAAc-PPy cryogel sheet at the top of the hydrogel substrate becomes short of water and dehydration makes the sheet gradually warp upwards. At the same time, the surface temperature of different cryogel sheets was measured every half an hour and the average values are demonstrated in Fig. S11a (ESI $\dagger$ ). After $30 \mathrm{~min}$, the temperatures for all the cryogel cases quickly rise to a level-off value with the highest for the PAAc-PPy@cryogel. Extraordinarily, the temperature of the PAAc-PPy@hydrogel rises to $45.3{ }^{\circ} \mathrm{C}$ at $150 \mathrm{~min}$, then keeps increasing up to $67.2{ }^{\circ} \mathrm{C}$ at $240 \mathrm{~min}$. In spite of the temperature increase in this case, the evaporation rate drops noticeably after $120 \mathrm{~min}$ (Fig. S11b, ESI $\dagger$ ).

Based on the above evaporation results, it is established that the PAAc cryogel as the substrate could efficiently transport
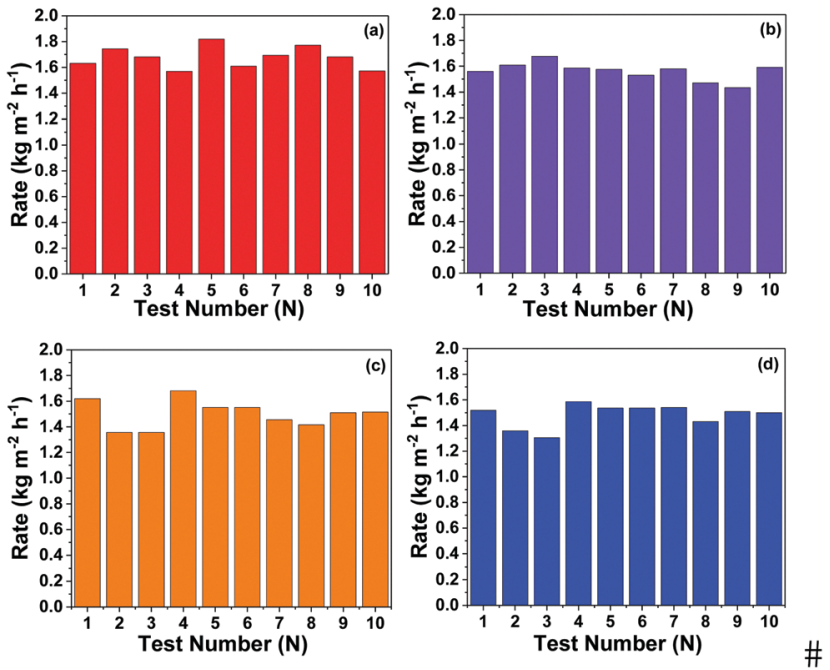

Fig. 7 Variations in the photothermal evaporation rate and efficiency with test number during three-month measurement (a: PAAc-PPy, b: PAAcPANI, c: PAAc-GO, d: PAAc-rGO cryogel sheet on the PAAc cryogel substrate) under one-sun irradiation for $60 \mathrm{~min}$.

water from the water reservoir to the evaporation sheet surface, while the PAAc hydrogel failed. The morphology observation by SEM, the measurement of the swelling rate, and the capillary test provide credible supports.

To clarify the long-term durability and reusability of the current photothermal evaporation system, different composite cryogel sheets and mushroom-shaped PAAc cryogel substrates have been assembled to test photothermal evaporation within three months. Each test was performed occasionally and the recorded durations were accidentally chosen. Fig. 7a-d show the results of ten tests for four composite cryogel sheets with the mushroom-shaped PAAc cryogel substrate. All the photothermal evaporation systems maintain a high evaporation rate.

\subsection{Photothermal evaporation of salt water with different cryogels}

To evaluate the application of cryogels in seawater desalination, simulated seawater $\left(3.0 \times 10^{4} \mathrm{mg} \mathrm{L}^{-1} \mathrm{Na}^{+}, 3.5 \times 10^{3} \mathrm{mg} \mathrm{L}^{-1}\right.$ $\mathrm{Mg}^{2+}$, and $1.76 \times 10^{3} \mathrm{mg} \mathrm{L}^{-1} \mathrm{~K}^{+}$) was used as the water reservoir to perform photothermal evaporation with the PAAc-PPy cryogel sheet and the PAAc cryogel substrate. Under one-sun irradiation, photothermal evaporation lasted for $12 \mathrm{~h}$. Then, the light was turned off and the tip of the mushroom-shaped cryogel maintained the same status for another $12 \mathrm{~h}$. Afterwards, this procedure was repeated in the following 4 days to follow the mass loss and salt precipitation on the cryogel sheet. Fig. 8a shows the mass loss at every one hour within the first day. Except an unreliable data point (at $10 \mathrm{~h}$ ), others are in linear correlation. Within each one-hour duration, the mass loss lies in the range of $1.343-1.648 \mathrm{~kg} \mathrm{~m}^{-2} \mathrm{~h}^{-1}$ and the average evaporation rate in the first $12 \mathrm{~h}$ is $1.560 \mathrm{~kg} \mathrm{~m}^{-2} \mathrm{~h}^{-1}$, which is a little lower than that of pure water. The decrease in the vapor pressure of the solution compared with that of the pure solvent is responsible for this result. As shown in Fig. 8b, the mass loss 

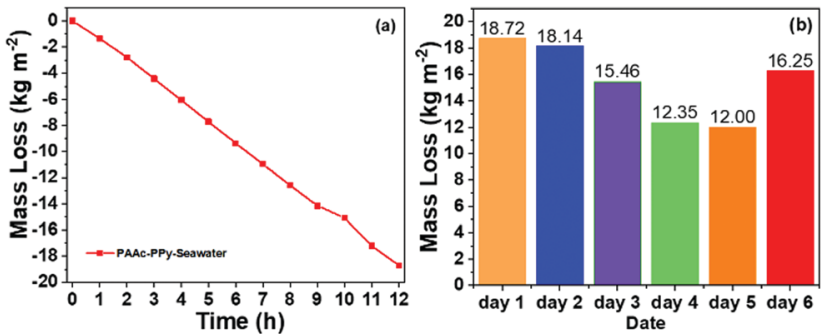

(c)

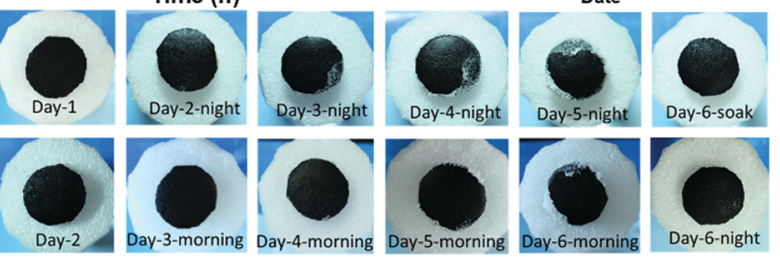

Fig. 8 Mass loss of the salty water reservoir at different hours of the first day (a) and at different days (b) under one-sun irradiation with the PAAcPPy cryogel sheet; (c) the surface appearance of the PAAc-PPy cryogel sheet at different time intervals.

of salty water reservoir in the following days keeps decreasing and exhibits a tendency of levelling-off at the fifth day. Considering water evaporation, the concentration of salty water keeps increasing, which is one reason for the further decrease in the evaporation rate. Another reason is the salting out during the evaporation, which might clog the pathway of water transport.

The surface appearance of the PAAc-PPy cryogel sheet was recorded, as shown in Fig. 8c. The salt separated out on the surface keeps increasing in amount at the night in the beginning of each day. It hinders the solar light absorption and water volatilization, and thus the evaporation rate of water might be reduced. However, due to rapid water transport inside the PAAc cryogel, some salt was dissolved during the night, which is evidenced by the less salt on the surface the next morning. At 20:00 pm of the fifth day, the salt on the surface of the PAAc-PPy cryogel reached the maximum amount due to the edgepreferential crystallization. ${ }^{46}$ Very little salt was dissolved up to 8:00 am of the sixth day. The mass loss of the salty water reservoir during the fifth day dropped to $12.00 \mathrm{~kg} \mathrm{~m}^{-2}$, being $64 \%$ of that during the first day. Therefore, this PAAc-PPy cryogel sheet and the PAAc cryogel substrate were immersed in simulated seawater to wash out the salt solid. Then, photothermal evaporation experiment was continued for an additional $12 \mathrm{~h}$. The cryogel surface looked neat without the salt solid both at morning and night beginning while the mass loss significantly increased to $16.25 \mathrm{~kg} \mathrm{~m}^{-2}$. In the practical performance of seawater desalination, the concentration of the seawater reservoir is constant, which is different from the increasing concentration of salty water reservoir in the current research. Thus, it is believed that the current photothermal evaporation system is convenient and efficient for seawater desalination.

With a self-made device (Fig. 9 right), photothermal evaporation of simulated seawater was performed under one-sun irradiation and the evaporated water was collected. As shown

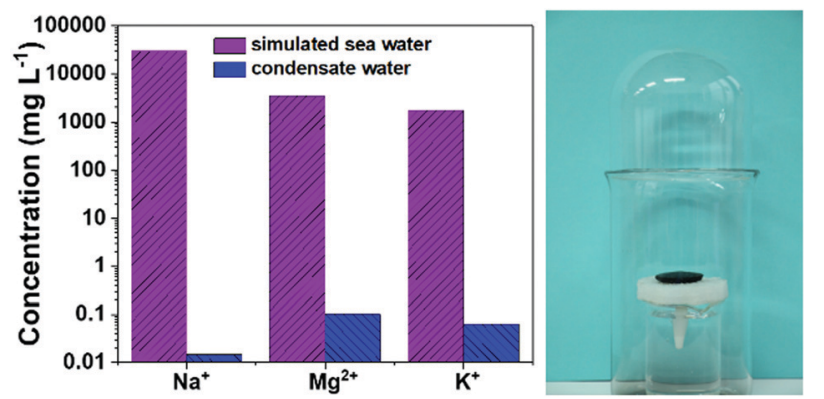

Fig. 9 The concentrations of the three ions in simulated seawater before and after photothermal desalination (left) with a self-made vapor collection device (right).

by the recorded video (ESI, $\uparrow$ Video S3), water vapor was generated under light irradiation and gradually condensed on the collector top. The water drops flowed along the inner surface and accumulated at the bottom. ICP-MS was used to determine the ion concentration. The ion concentrations of simulated seawater were pretty high. After photothermal evaporation, the concentrations of these ions were reduced by at least 4 orders of magnitude, which was $0.015 \mathrm{mg} \mathrm{L}^{-1}$ for $\mathrm{Na}^{+}, 0.102 \mathrm{mg} \mathrm{L}^{-1}$ for $\mathrm{Mg}^{2+}$, and $0.063 \mathrm{mg} \mathrm{L}^{-1}$ for $\mathrm{K}^{+}$(Fig. 9). All the ions are far below the fresh water taste threshold defined by the World Health Organization (WHO).

\section{Conclusions}

Through cryopolymerization, different PAAc cryogels with photothermal materials have been prepared to assemble photothermal evaporation systems with a composite cryogel sheet and a mushroom-shaped PAAc cryogel substrate. The hydrophilicity and hierarchical porosity facilitate rapid water absorption and efficient water transport compared with the PAAc hydrogel prepared at room temperature. The porous morphology is also helpful to lessen the heat loss. Due to the strong interaction between PAAc and the photothermal materials, composite cryogels with PPy, PANI, GO, and rGO exhibit uniform distribution of the photothermal materials inside the PAAc cryogel. Thus, the light absorbance of the composite cryogel sheet is almost $98 \%$ for the PAAc-PPy and PAAc-PANI cryogels. During steady photothermal evaporation under onesun irradiation, the evaporation rate of pure water for the PAAc-PPy cryogel sheet can reach $1.819 \mathrm{~kg} \mathrm{~m}^{-2} \mathrm{~h}^{-1}$ with an evaporation efficiency of about $100 \%$ if the possible heat loss and the deviation of evaporation enthalpy are neglected. However, the abnormal efficiency of the PAAc-GO sheet suggests the uncertainty of such an assumption. Increasing the incident light to four-sun, the evaporation rate increases significantly for all composite cryogel sheets. The PAAc-PPy cryogel sheet still exhibits the highest evaporation $\left(6.492 \mathrm{~kg} \mathrm{~m}^{-2} \mathrm{~h}^{-1}\right)$ but after replacing the substrate with the PAAc hydrogel, its evaporation rate drops noticeably $\left(2.962 \mathrm{~kg} \mathrm{~m}^{-2} \mathrm{~h}^{-1}\right)$ due to the poor water transport of the PAAc hydrogel. All photothermal evaporation systems with the composite cryogel sheet and the PAAc cryogel 
substrate demonstrate long-term availability and reproducibility of photothermal evaporation under one-sun irradiation but the PPAc-PPy sheet with the PAAc hydrogel substrate displays slower evaporation. As for the photothermal evaporation of salty water with the PPAc-PPy sheet with the PAAc hydrogel substrate, the evaporation rate under one-sun reaches $1.560 \mathrm{~kg} \mathrm{~m}^{-2} \mathrm{~h}^{-1}$ within the first $12 \mathrm{~h}$. The accumulated salt could be partially removed with renewed water through cryogel capillary and the performance was recovered by washing with salt water. The ion concentrations in condensed water reduced significantly. Therefore, the present PAAc cryogel is an excellent platform for photothermal evaporation to incorporate different active materials in water purification and seawater desalination.

\section{Author contributions}

Wei-Dong He: conceptualization, methodology, formal analysis, writing - reviewing and editing, supervision. Jia-Yun Wang: investigation, data curation, writing - original draft. Xiao-Xiao Guo: validation, writing - reviewing. Jun Chen: formal analysis, visualization. Investigation - some experiments, validation. Hui-Juan Li: formal analysis, resource, investigation - some experiments. Abdul Haleem and Sheng-Qi Chen: writing teviewing and editing.

\section{Conflicts of interest}

There are no conflicts to declare.

\section{Acknowledgements}

The authors are grateful for the financial support provided by National Natural Science Foundation of China (20934005 and 21274136), Chinese Academy of Sciences President's International Fellowship Initiative (2017VEA0031) and Natural Science Foundation of Anhui Province (1808085QE125). Abdul Haleem wishes to acknowledge Chinese Scholarship Council for his scholarship of PhD studies.

\section{References}

1 Y. Jiang, J. Environ. Manage., 2009, 90(11), 3185-3196.

2 R. P. Schwarzenbach, T. Egli, T. B. Hofstetter, U. von Gunten and B. Wehrli, Annu. Rev. Environ. Resour., 2010, 35(1), 109-136.

3 Q. Gan, T. Zhang, R. Chen, X. Wang and M. Ye, ACS. Sustainable Chem. Eng., 2019, 7(4), 3925-3932.

4 X. Wu, M. E. Robson, J. L. Phelps, J. S. Tan, B. Shao and G. Owens, et al., Nano Energy, 2019, 56, 708-715.

5 Y. Xu, J. Ma, D. Liu, H. Xu, F. Cui and W. Wang, Chem. Eng. J., 2019, 356, 869-876.

6 V. Kashyap, A. Al-Bayati, S. M. Sajadi, P. Irajizad, S. H. Wang and H. Ghasemi, J. Mater. Chem. A, 2017, 5(29), 15227-15234.

7 Y. Li, T. Gao, Z. Yang, C. Chen, W. Luo and J. Song, et al., Adv. Mater., 2017, 29(26), 1700981.
8 Y. Lin, H. Xu, X. Shan, Y. Di, A. Zhao and Y. Hu, et al., J. Mater. Chem. A, 2019, 7(33), 19203-19227.

9 G. Liu, J. Xu and K. Wang, Nano Energy, 2017, 41, 269-284.

10 M. Gao, L. Zhu, C. K. Peh and G. W. Ho, Energy Environ. Sci., 2019, 12(3), 841-864.

11 B. Zhu, H. Kou, Z. Liu, Z. Wang, D. K. Macharia and M. Zhu, et al., ACS. Appl. Mater. Interfaces, 2019, 11(38), 35005-35014.

12 X. Wu, T. Gao, C. Han, J. Xu, G. Owens and H. Xu, Sci. Bull., 2019, 64(21), 1625-1633.

13 F. Gong, W. Wang, H. Li, D. Xia, Q. Dai and X. Wu, et al., Appl. Energy, 2020, 261, 114410.

14 H. Wang, A. Du, X. Ji, C. Zhang, B. Zhou and Z. Zhang, et al., ACS. Appl. Mater. Interfaces, 2019, 11(45), 42057-42065.

15 W. Li, Z. Li, K. Bertelsmann and D. E. Fan, Adv. Mater., 2019, 31(29), e1900720.

16 Q. Chen, Z. Pei, Y. Xu, Z. Li, Y. Yang and Y. Wei, et al., Chem. Sci., 2018, 9(3), 623-628.

17 Z. Wang, Y. Yan, X. Shen, C. Jin, Q. Sun and H. Li, J. Mater. Chem. A, 2019, 7(36), 20706-20712.

18 W. Wang, J. Niu, J. Guo, L. Yin and H. Huang, Sol. Energy Mater. Sol. C, 2019, 201, 110046.

19 H.-Y. Zhao, J. Zhou, Z.-L. Yu, L.-F. Chen, H.-J. Zhan and H.-W. Zhu, et al., Cell Rep. Phys. Sci., 2020, 1(6), 100074.

20 Q. Wang, L. Qiu, Y. Jia, Y. Chang, X. Tan and L. Yang, et al., Sol. Energy Mater. Sol. C, 2019, 202, 110116.

21 Y. Chen, Y. Shi, H. Kou, D. Liu, Y. Huang and Z. Chen, et al., ACS Sustainable Chem. Eng., 2019, 7(3), 2911-2915.

22 D.-D. Qin, Y.-J. Zhu, F.-F. Chen, R.-L. Yang and Z.-C. Xiong, Carbon, 2019, 150, 233-243.

23 C. Zhang, B. Yuan, Y. Liang, L. Yang, L. Bai and H. Yang, et al., Mater. Chem. Phys., 2021, 258, 123998.

24 F. Zhao, X. Zhou, Y. Shi, X. Qian, M. Alexander and X. Zhao, et al., Nat. Nanotechnol., 2018, 13(6), 489-495.

25 D. Weng, F. Xu, X. Li, Y. Li and J. Sun, J. Mater. Chem. A, 2018, 6(47), 24441-24451.

26 L. Zhu, M. Gao, C. K. N. Peh, X. Wang and G. W. Ho, Adv. Energy Mater., 2018, 8(16), 1702149.

27 J. Hou, S. Liu, Y. Ning, Y. Wang, Y. Yang and Q. Wang, J. Alloys Compd., 2021, 851, 156879.

28 C. Xiao, W. Liang, Q.-M. Hasi, L. Chen, J. He and F. Liu, et al., Mater. Today Energy, 2020, 16, 100417.

29 B. Yuan, C. Zhang, Y. Liang, L. Yang, H. Yang and L. Bai, et al., Adv. Sustainable Syst., 2020, 2000245.

30 N. Ma, Q. Fu, Y. Hong, X. Hao, X. Wang and J. Ju, et al., ACS Appl. Mater. Interfaces, 2020, 12(15), 18165-18173.

31 Q. F. Guan, Z. M. Han, Z. C. Ling, H. B. Yang and S. H. Yu, Nano Lett., 2020, 5699-5704.

32 J. Tang, T. Zheng, Z. Song, Y. Shao, N. Li and K. Jia, et al., ACS Appl. Mater. Interfaces, 2020, 12(16), 18504-18511.

33 M. Zhu, Y. Li, F. Chen, X. Zhu, J. Dai and Y. Li, et al., Adv. Energy Mater., 2018, 8(4), 1701028.

34 L. Shi, Y. Wang, L. Zhang and P. Wang, J. Mater. Chem. A, 2017, 5(31), 16212-16219.

35 N. Xu, X. Hu, W. Xu, X. Li, L. Zhou and S. Zhu, et al., Adv. Mater., 2017, 29(28), 1606762. 
36 H. Kou, Z. Liu, B. Zhu, D. K. Macharia, S. Ahmed and B. Wu, et al., Desalination, 2019, 462, 29-38.

37 H. Huang, J. Yao, L. Li, F. Zhu, Z. Liu and X. Zeng, et al., J. Mater. Sci., 2016, 51(18), 8728-8736.

38 Y. Wang, L. Zhang and P. Wang, ACS Sustainable Chem. Eng., 2016, 4(3), 1223-1230.

39 L. Zhang, B. Tang, J. Wu, R. Li and P. Wang, Adv. Mater., 2015, $27(33)$, 4889-4894.

40 C.-S. Hu, H.-J. Li, J.-Y. Wang, A. Haleem, X.-C. Li and M. Siddiq, et al., ACS. Appl. Energy Mater., 2019, 2(10), 7554-7563.
41 K. D. V. Marcano and D. C. Berlin, et al., ACS Nano, 2010, 4(8), 4806.

42 N. Sahiner and F. Seven, Energy, 2014, 71, 170-179.

43 R. Sun, H. Chen, Q. Li, Q. Song and X. Zhang, Nanoscale, 2014, 6(21), 12912-12920.

44 J. Zhang, H. Yang, G. Shen, P. Cheng, J. Zhang and S. Guo, Chem. Commun., 2010, 46(7), 1112-1114.

45 Y. Guo, X. Zhao, F. Zhao, Z. Jiao, X. Zhou and G. Yu, Energy Environ. Sci., 2020, 13(7), 2087-2095.

46 Y. Xia, Q. Hou, H. Jubaer, Y. Li, Y. Kang and S. Yuan, et al., Energy Environ. Sci., 2019, 12(6), 1840-1847. 